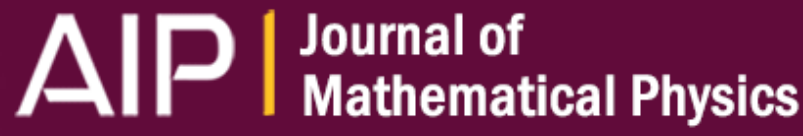

Chern-Simons action for inhomogeneous Virasoro group as extension of three dimensional flat gravity

Glenn Barnich, Gastón Giribet, and Mauricio Leston

Citation: Journal of Mathematical Physics 56, 071701 (2015); doi: 10.1063/1.4926456

View online: http://dx.doi.org/10.1063/1.4926456

View Table of Contents: http://scitation.aip.org/content/aip/journal/jmp/56/7?ver=pdfcov

Published by the AIP Publishing

\section{Articles you may be interested in}

Extension of Chern-Simons forms

J. Math. Phys. 55, 062304 (2014); 10.1063/1.4882086

All the solutions of the form M $2 \times \mathrm{W} \Sigma \mathrm{d}-2$ for Lovelock gravity in vacuum in the Chern-Simons case

J. Math. Phys. 54, 042501 (2013); 10.1063/1.4795258

Dirac matrices for Chern-Simons gravity

AIP Conf. Proc. 1471, 118 (2012); 10.1063/1.4756824

Gravitational Chern-Simons and the adiabatic limit

J. Math. Phys. 51, 122502 (2010); 10.1063/1.3528667

Existence of the self-graviting Chern-Simons vortices

J. Math. Phys. 44, 5616 (2003); 10.1063/1.1625871

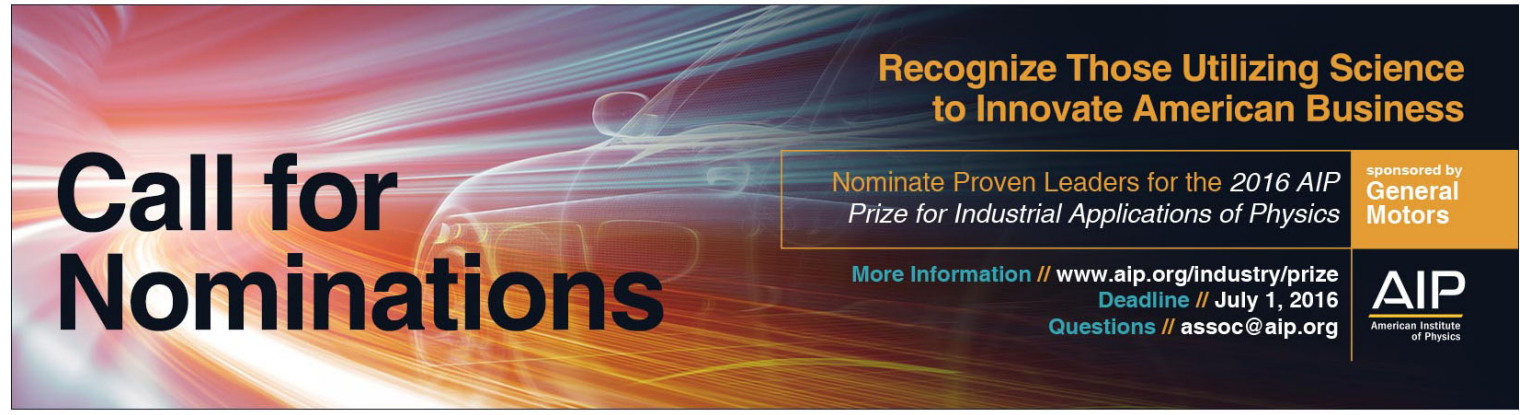




\title{
Chern-Simons action for inhomogeneous Virasoro group as extension of three dimensional flat gravity
}

\author{
Glenn Barnich, ${ }^{1}$ Gastón Giribet, ${ }^{1,2,3}$ and Mauricio Leston ${ }^{4}$ \\ ${ }^{1}$ Physique Théorique et Mathématique, Université Libre de Bruxelles and International \\ Solvay Institutes, Campus Plaine C.P. 231, B-1050 Bruxelles, Belgium \\ ${ }^{2}$ Universidad de Buenos Aires FCEN-UBA and IFIBA-CONICET, Ciudad Universitaria, \\ Pabellón I, 1428 Buenos Aires, Argentina \\ ${ }^{3}$ Instituto de Física, Pontificia Universidad Católica de Valparaíso, Casilla 4059, \\ Valparaíso, Chile \\ ${ }^{4}$ Instituto de Astronomía y Física del Espacio IAFE-CONICET, Ciudad Universitaria, \\ Pabellón IAFE, C.C. 67 Suc. 28, 1428 Buenos Aires, Argentina
}

(Received 13 May 2015; accepted 27 June 2015; published online 10 July 2015)

\begin{abstract}
We initiate the study of a Chern-Simons action associated to the semi-direct sum of the Virasoro algebra with its coadjoint representation. This model extends the standard Chern-Simons formulation of three dimensional flat gravity and is similar to the higher-spin extension of three dimensional anti-de Sitter or flat gravity. The extension can also be constructed for the exotic but not for the cosmological constant deformation of flat gravity. @ 2015 AIP Publishing LLC. [http://dx.doi.org/10.1063/1.4926456]
\end{abstract}

\section{INTRODUCTION}

Higher spin extensions of $\mathrm{AdS}_{3}$ gravity ${ }^{1}$ have attracted a lot of attention recently (see, e.g., Refs. 2-6) as they allow one to probe novel aspects of the anti-de Sitter/conformal field theory (AdS/CFT) correspondence beyond the purely gravitational sector in the most tractable setting of three bulk dimensions. More recently, such higher spin extensions have also been constructed for three dimensional flat gravity. ${ }^{7,8}$

A key feature of the Chern-Simons formulation of pure gravity in flat space ${ }^{9,10}$ is the fact that the action does not involve the Killing metric like it does for semi-simple Lie algebras, but the non-degenerate invariant pairing that exists between the translation and the rotation generators in three dimensions. More generally, such a metric, and thus also a Chern-Simons model, exists for all algebras $\mathfrak{g} \ltimes_{\mathrm{ad}^{*}} \mathfrak{g}^{*}$ that are the semi-direct sum of a given algebra $\mathfrak{g}$ with its coadjoint representation $\mathrm{g}^{*}$ embedded as an abelian ideal. ${ }^{11,12}$

Since the Lorentz algebra $\mathfrak{s} \mathfrak{D}(2,1)$ is isomorphic to $\mathfrak{s l}(2, \mathbb{R})$, probably the most obvious infinitedimensional extension of flat gravity consists in replacing $\mathfrak{s l}(2, \mathbb{R})$ by the Virasoro algebra vir. Such a Chern-Simons Virasoro model involves all ingredients that are used when applying the orbit method to the study of representations of the Virasoro algebra (see, e.g., Refs. 13-16 for considerations in the physics literature). One might hope that the model can be useful in this context, in the same way as the Poisson sigma model ${ }^{17,18}$ is relevant to the problem of quantizing Poisson manifolds. ${ }^{19,20}$

A further motivation to study the model comes from the representation theory of the symmetry algebra of asymptotically flat three dimensional spacetimes ${ }^{21}$ (see also Ref. 22). This algebra is given by the Bondi-Metzner-Sachs (BMS) algebra in three dimensions, which is the semi-direct sum of the algebra of vector fields on the circle with its adjoint representation embedded as an abelian ideal, $\mathfrak{b m s}_{3}=\operatorname{Vect}\left(S^{1}\right) \ltimes_{\mathrm{ad}} \operatorname{Vect}\left(S^{1}\right)_{\mathrm{ab}}$. Because of theorems available on unitary irreducible representations of finite dimensional Lie algebras and groups with this particular structure (see, e.g., Ref. 23), it is natural to study induced representations, for which it is again the coadjoint representation of the Virasoro algebra with its orbits and little groups that is relevant. ${ }^{24}$ Finally, the solution space of three-dimensional asymptotically anti-de Sitter spacetimes is also classified by (two copies of) the coadjoint orbits of the Virasoro group (see, e.g., Refs. 25 and 26 for recent discussions). In the flat case, the coadjoint orbits of the $\mathrm{BMS}_{3}$ group are controlled by Virasoro coadjoint orbits in a standard way appropriate to semi-direct products. ${ }^{27}$ 
After reviewing the formulation of Chern-Simons theories for inhomogeneous groups, we start our analysis of the model with a detailed discussion of how flat gravity and its solutions are included in this infinite-dimensional extension. We will show that gravity solutions can be included in the extended model at the price of turning on additional non-gravity modes that, however, do not backreact on the geometry.

We then study its consistent deformations. This is most conveniently done in the context of the Batalin-Vilkovisky (BV) antifield formalism ${ }^{28-32}$ (see also Refs. 33 and 34 for reviews) where they are controlled by the cohomology of the BV differential in the space of local functionals in ghost number $0^{35}$ (see also Ref. 36). In particular, in the case of $i \mathfrak{s} \mathfrak{D}(2,1)$, the deformations provided in Ref. 10 have been studied from this point of view in Ref. 37, Sec. 7. More generally, in any ghost number, the BV cohomology in the space of local functionals is locally isomorphic to the Chevalley-Eilenberg cohomology of the Lie algebra with which the Chern-Simons theory is constructed. This is a particular case of a general result valid for AKSZ sigma models ${ }^{38}$ for which the cohomology of the BV differential in the space of local functionals is locally isomorphic to the cohomology of the target space differential. ${ }^{39}$ An interesting feature is that the other primitive generator in degree 3 of the cohomology ring of Vect $\left(S^{1}\right)$ appears in this deformation.

We end with considerations on the dual boundary theory. Chern-Simons theories on a solid cylinder induce chiral Wess-Zumino-Witten theories on their boundary. ${ }^{40,41}$ For the Chern-Simons formulation of three dimensional gravity, the boundary theory has been studied in the flat case in Ref. 42, in the anti-de Sitter case with non-trivial asymptotics in Ref. 43, and in the flat case with non-trivial asymptotics at null infinity in Ref. 44. For the Chern-Simons Virasoro model, one ends up with a chiral Wess-Zumino model with a current algebra determined by $\mathfrak{v i r} \ltimes_{\mathrm{ad}^{*}} \mathfrak{p i r}^{*}$. A classical Sugawara construction then manifestly shows that the model is conformally invariant.

\section{CHERN-SIMONS MODEL FOR INHOMOGENEOUS GROUPS}

The Chern-Simons formulation of three dimensional flat gravity is based on the Poincare algebra $i \mathfrak{s o}(2,1)$. If the generators are denoted by $P_{a}, J_{b}$, the invariant inner product required to construct the action is given by $\left\langle P_{a}, J_{b}\right\rangle=\eta_{a b},\left\langle P_{a}, P_{b}\right\rangle=0=\left\langle J_{a}, J_{b}\right\rangle$ with $\eta_{a b}$ the Minkowski metric in three dimensions. This construction can be generalized by replacing $\mathfrak{s p}(2,1)$ with a generic Lie algebra $\mathrm{g}$ : the total Lie algebra is the semi-direct sum of $\mathfrak{g}$, whose generators are denoted by $e_{a}$, with its coadjoint representation embedded as an abelian ideal,

$$
\left[e_{a}, e_{b}\right]=f_{a b}^{c} e_{c}, \quad\left[e_{a}, e^{* b}\right]=-f_{a c}^{b} e^{* c}, \quad\left[e^{* a} e^{* b}\right]=0 .
$$

The reason why it is always possible to construct a Chern-Simons action for such a Lie algebra is the existence of the non-degenerate invariant inner product,

$$
\left\langle e_{a}, e^{* b}\right\rangle=\delta_{a}^{b}, \quad\left\langle e_{a}, e_{b}\right\rangle=0=\left\langle e^{* a}, e^{* b}\right\rangle .
$$

\section{CHERN-SIMONS VIRASORO MODEL}

What we want to do here is use as Lie algebra g the Virasoro algebra vir with generators $L_{m}, Z$,

$$
\left[L_{m}, L_{n}\right]=(m-n) L_{m+n}+\frac{Z}{12} \delta_{m+n}^{0} m\left(m^{2}-1\right), \quad\left[L_{m}, Z\right]=0,
$$

so that

$$
\begin{aligned}
& {\left[L_{m}, L^{* n}\right]=(n-2 m) L^{* n-m}, \quad\left[L_{m}, Z^{*}\right]=-\frac{1}{12} m\left(m^{2}-1\right) L^{*-m},} \\
& {\left[Z, L^{* n}\right]=0=\left[Z, Z^{*}\right], \quad\left[L^{* m}, L^{* n}\right]=0=\left[L^{* m}, Z^{*}\right] .}
\end{aligned}
$$

If the associated gauge field is denoted by $A=A_{\mu}^{A} T_{A} d x^{\mu}$, with $x^{\mu}$ local coordinates on a threedimensional manifold $\mathcal{M}_{3}$ and $T_{A}=\left(L_{m}, Z, L^{* m}, Z^{*}\right)$, the Chern-Simons action is

$$
S_{0}[A]=\kappa \int_{\mathcal{M}_{3}} \frac{1}{2}\left\langle A, d A+\frac{2}{3} A^{2}\right\rangle .
$$


In more details, let $A=A^{m} L_{m}+D Z+B_{m} L^{* m}+C Z^{*}$. The associated curvatures are

$$
\begin{aligned}
& F^{m}=d A^{m}+\frac{1}{2} f_{n k}^{m} A^{n} A^{k}, \quad F^{Z}=d D+\frac{1}{24} m\left(m^{2}-1\right) A^{-m} A^{m}, \\
& F_{m}=d B_{m}-f_{n m}^{k} A^{n} B_{k}+\frac{1}{12} m\left(m^{2}-1\right) A^{-m} C, \quad F_{Z^{*}}=d C,
\end{aligned}
$$

where $f_{m n}^{k}=(m-n) \delta_{m+n}^{k}$. The equations of motions are equivalent to requiring these curvatures to vanish.

This theory can also be understood as a three dimensional BF-type theory for the Virasoro algebra. Indeed, through integrations by parts, action (3.3) can be rewritten as

$$
S_{0}=\kappa \int_{\mathcal{M}_{3}}\left(B_{m} F^{m}+C F^{Z}\right) .
$$

Finally, introducing an additional circle $S^{1}$ with coordinate $\phi \in[0,2 \pi)$, elements of vir are pairs $(v,-i a)$ with $v=f(\phi) \partial_{\phi}$ vector fields on the circle and $a$ a real number. In particular, $L_{m}=\left(i e^{i m \phi} \partial_{\phi}, 0\right)$ for $m \neq 0, L_{0}=\left(i \partial_{\phi}, \frac{1}{24}\right), Z=(0,1)$. The commutation relations are given by

$$
\left[\left(v_{1},-i a_{1}\right),\left(v_{2},-i a_{2}\right)\right]=\left(\left(f_{1} f_{2}^{\prime}-f_{2} f_{1}^{\prime}\right) \partial_{\phi},-\frac{i}{48 \pi} \int_{0}^{2 \pi} d \phi\left(f_{1}^{\prime} f_{2}^{\prime \prime}-f_{2}^{\prime} f_{1}^{\prime \prime}\right)\right) .
$$

Coadjoint vectors are given by pairs $(u, i t)$, where $u=h(\phi) d \phi^{2}$ is a quadratic differential and $t$ a real number. In these terms, the invariant inner product is given by

$$
\langle(u, i t),(v,-i a)\rangle=\int_{0}^{2 \pi} d \phi h f+a t,
$$

with coadjoint action

$$
a d_{(f,-i a)}^{*}(u, i t)=\left(\left(f h^{\prime}+2 f^{\prime} h-\frac{t}{24 \pi} f^{\prime \prime \prime}\right) d \phi^{2}, 0\right) .
$$

In particular, $L^{* m}=\left(-\frac{i e^{-i m \phi}}{2 \pi}, 0\right), Z^{*}=\left(\frac{i}{48 \pi}, 1\right)$.

Let $(\mathcal{A},-i D)$ and $(\mathcal{B}, i C)$ be one forms on $\mathcal{M}_{3}$ with values in vir, respectively, vir* and let $\left(\mathcal{F},-i F^{Z}\right)=d(\mathcal{A},-i D)+\frac{1}{2}[(\mathcal{A},-i D),(\mathcal{A},-i D)]$. Instead of an infinite sum over $m$, Chern-Simons action (3.5) can then be written with an additional integral over $\phi$ as

$$
S_{0}=\kappa \int_{\mathcal{M}_{3}}\left\langle(\mathcal{B}, i C),\left(\mathcal{F},-i F^{Z}\right)\right\rangle
$$

and interpreted as a field theory on $\mathcal{M}_{3} \times S^{1}$.

Interestingly, the construction of Chern-Simons theories for inhomogeneous groups as described in Section III can also be done by using as $\mathfrak{g}$ the semi-direct sum of the Virasoro algebra with the affine Kac-Moody algebra for $\mathfrak{s l}(2, \mathbb{R})$. A Chern-Simons theory of precisely this type (but without the Virasoro central extension) has appeared previously in the context of a Kaluza-Klein reduction for four dimensional gravity on a circle in Refs. 45 and 46.

\section{EXTENSION OF FLAT GRAVITY}

The Virasoro Chern-Simons model may be interpreted as describing the non-trivial coupling of an infinite number of additional gauge fields to gravity in three dimensions since the action reduces to the Einstein-Hilbert one by putting to zero the gauge fields associated with the generators $Z, Z^{*}$ and $L_{m}, L^{* m}$ for $m \neq-1,0,1$. Indeed, when all gauge fields besides $A^{m}, B_{m}, m,=-1,0,1$ are switched off, we get

$$
S_{0}=\kappa \int B_{m} F^{m}, \quad m, n, k \in-1,0,1 .
$$

The standard gravitational formulation is recovered through a change of basis: $A^{m} L_{m}+B_{m} L^{* m}=$ $\omega^{a} J_{a}+e^{a} P_{a}$, with $a, b=0,1,2, J^{a}=-\frac{1}{2} \epsilon^{a b c} J_{b c},\left[J_{a}, J_{b}\right]=\epsilon_{a b c} J^{c},\left[J_{a}, P_{b}\right]=\epsilon_{a b c} P^{c},\left[P_{a}, P_{b}\right]=$ 
$0, \epsilon_{012}=1$, where $\omega^{a}$ and $e^{a}$ are the spin connection and vielbein 1-forms, respectively. Indices are lowered and raised with $\eta_{a b}=\operatorname{diag}(-1,1,1)$ and its inverse while

$$
\begin{aligned}
& l_{-1}=J_{0}-J_{1}, \quad l_{1}=J_{0}+J_{1}, \quad l_{0}=-J_{2}, \\
& P_{0}=-l^{* 1}-l^{*-1}, \quad P_{1}=l^{* 1}-l^{*-1}, \quad P_{2}=-l^{* 0},
\end{aligned}
$$

and action (4.1) coincides with the Einstein-Hilbert action in terms of dreibeins and spin connections,

$$
S_{0}[e, \omega]=\frac{1}{16 \pi G} \int e e_{a}^{\mu} e_{b}^{v} R_{\mu \nu}^{a b} d^{3} x
$$

provided $\kappa=-\frac{1}{8 \pi G}$.

The question is then whether any solution to the gravity equations of motions can be lifted to a solution of the extended system. More generally, consider a Chern-Simons theory based on $\mathfrak{g} \ltimes_{\mathrm{ad}^{*}} \mathfrak{g}^{*}$ and let $\mathfrak{h} \subset \mathfrak{g}$ be a subalgebra. In the gravity case, $\mathfrak{h}=\mathfrak{s l}(2, \mathbb{R})$, while $\mathfrak{g}=$ vir. Can any solution of the Chern-Simons theory based on $\mathfrak{h} \ltimes_{\mathrm{ad}^{*}} \mathfrak{h}^{*}$ be lifted to a solution of the Chern-Simons theory based on $\mathfrak{g} \ltimes_{\mathrm{ad}^{*}} \mathfrak{g}^{*}$ ?

Answering this question is not completely trivial because $\mathfrak{h} \ltimes_{a^{*}} \mathfrak{h}^{*}$ is not a subalgebra of $\mathfrak{g} \ltimes_{\mathrm{ad}^{*}} \mathfrak{g}^{*}$. Indeed, let us denote by $e_{\gamma}$ generators of $\mathfrak{h}$ and by $e_{\Gamma}=\left(e_{\gamma}, e_{C}\right)$ generators of $\mathfrak{g}$. The equations of motion of the theory based on $g$ are

$$
d A^{\Gamma}+\frac{1}{2} f_{\Delta \Sigma}^{\Gamma} A^{\Delta} A^{\Sigma}=0, \quad d B_{\Gamma}-f_{\Sigma \Gamma}^{\Delta} A^{\Sigma} B_{\Delta}=0,
$$

while those of the theory based on $\mathfrak{h}$ are similar but with upper case Greek indices replaced by lower case ones. Now, while it is true that given a solution of the theory based on $\mathfrak{h}$, the first set of equations in (4.4) can always be solved by setting to zero the all gauge fields $A^{C}$ since $f_{\gamma \delta}^{S}=0$; this cannot be done for the second set of equations since switching off all fields $B_{C}$ results in the additional constraints $f_{\delta C}^{\gamma} A^{\delta} B_{\gamma}=0$ on the fields of the theory based on $\mathfrak{h}$.

Let us then provide a formal argument showing that every solution of the theory based on $\mathfrak{h}$ can be lifted to a solution of the theory based on $\mathfrak{g}$ by keeping the fields $B_{\gamma}$ unchanged and suitably turning on additional fields $B_{C}$. Indeed, locally, the general solution of the theory based on $\mathfrak{h}$ is given by

$$
A=h^{-1} d h, \quad B=h^{-1}\left(d C_{\gamma} e^{* \gamma}\right) h,
$$

with $h$ a group element associated to $\mathfrak{h}$ and $C_{\gamma}(x)$ the arbitrary spacetime-dependent functions. The lift of the solution is then simply obtained by considering the formal group element $h=\mathrm{e}^{k^{\alpha}} e_{\alpha}$ with spacetime dependent functions $k^{\alpha}(x)$ to be an element of the group associated to $g$. This gives

$$
B=d C_{\gamma} \mathrm{e}^{-\left[k^{\alpha} e_{\alpha}, \cdot\right]} e^{* \gamma}=d C_{\gamma}\left(e^{* \gamma}+k^{\alpha} f_{\alpha \Delta}^{\gamma} e^{* \Delta}+\frac{1}{2} k^{\alpha} k^{\beta} f_{\beta \Sigma}^{\gamma} f_{\alpha \Delta}^{\Sigma} e^{* \Delta}+\cdots\right) .
$$

The reason why $B_{\gamma}$ is unchanged is that

$$
\left.\left[\mathfrak{h},\left[\mathfrak{h}, \ldots\left[\mathfrak{h}, \mathfrak{g}^{*}\right] \ldots\right]\right]\right|_{h^{*}}=\left[\mathfrak{h},\left[\mathfrak{h}, \ldots\left[\mathfrak{h}, \mathfrak{h}^{*}\right] \ldots\right]\right]_{\mathfrak{h} \propto_{\mathrm{ad}^{*}} \mathfrak{h}^{*}}
$$

since $\mathfrak{h}$ is a subalgebra of $\mathfrak{g}$.

Note however that the exponential map does not define a local chart close to the identity for the diffeomorphism of the circle or the Virasoro group. The above argument then only makes sense if one thinks about these groups formally, as done in the current context for instance in Refs. 47 and 15.

More concretely, let us consider for instance the general solution of three-dimensional flat gravity in BMS gauge. ${ }^{48}$ The associated vielbein and spin connections are given in Ref. 44. Explicitly, when taking into account (4.2), they translate to

$$
\begin{array}{r}
A^{ \pm 1}=\left(\frac{M}{2} \mp 1\right) d \phi, \quad A^{0}=0, \\
B_{ \pm 1}=-\frac{1}{2}\left(\left(\frac{M}{2} \pm 1\right) d u-d r+N d \phi\right), \quad B_{0}=-r d \phi .
\end{array}
$$


Here, $M$ and $N$ are defined in terms of two arbitrary function of $\phi, \Theta(\phi)$ and $\Xi(\phi)$ through $M=\Theta$, $N=\Xi+\frac{u}{2} \Theta^{\prime}$.

According to the previous considerations, we can assume $A^{m}=0=D$ for $|m|>1$. Let us then show that it is enough to turn on in addition $B_{ \pm 2}$ in order to extend the above solutions.

Indeed, if in addition $B_{m}=0=C$ for $|m|>2$, the equations for $d B_{m}, d C$ are automatically fulfilled for $|m|>3$. The equations for $|m|=2,3$ read explicitly

$$
\begin{aligned}
d B_{2}+3 A^{-1} B_{1}+A^{1} B_{3}+2 A^{0} B_{2} & =0, \\
d B_{-2}-3 A^{1} B_{-1}-A^{-1} B_{-3}-2 A^{0} B_{-2} & =0 \\
A^{-1} B_{2}=0, \quad A^{1} B_{-2} & =0 .
\end{aligned}
$$

Choosing $B_{2}=f_{+} A^{-1}$ and $B_{-2}=f_{-} A^{1}$ for some functions $f_{+}$and $f_{-}$allows one to satisfy the last 2 equations, while the first 2 turn into a system of partial differential equations for $f_{+}$and $f_{-}$,

$$
\begin{aligned}
& d\left(f_{+} A^{-1}\right)+3 A^{-1} B_{1}+2 f_{+} A^{0} A^{1}=0, \\
& d\left(f_{-} A^{1}\right)-3 A^{1} B_{-1}-2 f_{-} A^{0} A^{-1}=0 .
\end{aligned}
$$

By using that for (4.7), $A_{0}=0, d A^{ \pm 1}=0$, they reduce to $d f_{ \pm}=\mp 3 B_{ \pm 1}$. The associated integrability conditions $d B^{ \pm 1}=0$ are satisfied for (4.7) and

$$
f_{ \pm}= \pm \frac{3}{2}\left(\frac{u}{2} \Theta \pm u+\widetilde{\Xi}-r\right)
$$

where $\widetilde{\Xi}^{\prime}=\Xi$.

Then, we see explicitly that the field equations for the gravity modes are indeed satisfied. The gravity modes, even when they source the extra modes with $|m|>1$, do it in such a way that the latter, if originally switched off, do not backreact on Einstein's field equations.

\section{DEFORMATIONS OF THE CHERN-SIMONS VIRASORO MODEL}

Studying consistent deformations of Chern-Simons action (3.3) in the context of the BatalinVilkovisky antifield formalism involves several steps.

(i) All infinitesimal deformations must belong to the cohomology group $H^{0,3}(s \mid d)$, where $s$ is the antifield dependent Becchi-Rouet-Stora-Tyutin (BRST) differential associated with the Batalin-Vilkovisky master action for Virasoro Chern-Simons action (3.3).

(ii) For Chern-Simons theories, $H^{0,3}(s \mid d)$ is isomorphic to the Chevalley-Eilenberg cohomology of the associated Lie algebra.

For simplicity, let us first restrict ourselves to the non-centrally extended case of the Lie algebra witt $\ltimes$ witt $^{*}$ with generators $l_{m}, l^{* n}$, so that $c, Z, Z^{*}=0$. In this case, $H^{0,3}(s \mid d) \cong H^{3}($ witt $\ltimes$ witt* $)$.

(iii) The Hochschild-Serre analysis ${ }^{49}$ can be adapted to the current problem. Denoting the generators of $\wedge\left(\mathfrak{w i t t} \ltimes \mathfrak{w i t t}^{*}\right)^{*}$ by $\eta^{m}$ and $C_{m}$, the Chevalley-Eilenberg coboundary operator can be written as

$$
\gamma=-\frac{1}{2} \eta^{m} \eta^{n} f_{m n}^{k} \frac{\partial}{\partial \eta^{k}}+\eta^{m} \rho\left(l_{m}\right), \quad \rho\left(l_{m}\right)=f_{m n}^{k} C_{k} \frac{\partial}{\partial C_{n}} .
$$

Let $N_{\eta}=\eta^{m} \frac{\partial}{\partial \eta^{m}}$ and $N_{C}=C_{m} \frac{\partial}{\partial C_{m}}$. Since $\left[\gamma, N_{C}\right]=0$, the problem decomposes into separate cohomology problems with eigenvalues $(3,0),(2,1),(1,2),(0,3)$ for $\left(N_{\eta}, N_{C}\right)$.

In degree (3,0), there is but one cohomology class (Refs. 50 and 51, Theorem 2.4.2), which can be written as $\beta=\frac{1}{3 !} \beta_{m n k} \eta^{m} \eta^{n} \eta^{k}$ with

$$
\beta_{m n k}=\beta_{[m n k]}=\delta_{m+n+k}^{0}(m n(m-n)+n k(n-k)+m k(k-m)) .
$$


If the gauge field is denoted by $B^{m} l_{m}+A_{m} l^{* m}$, the $\mathrm{BV}$ master action is given by

$$
\begin{aligned}
& S=\kappa \int[ A_{m} d B^{m}+\frac{1}{2} A_{k} f_{m n}^{k} B^{m} B^{n}+ \\
&+\star A^{* m}\left(d C_{m}+f_{m n}^{k} B^{n} C_{k}-f_{m n}^{k} A_{k} \eta^{n}\right)+\star B_{m}^{*}\left(d \eta^{m}+f_{n k}^{m} B^{n} \eta^{k}\right)+ \\
&\left.\quad+\frac{1}{2} \star \eta_{k}^{*} f_{m n}^{k} \eta^{m} \eta^{n}-\star C^{* m} f_{m n}^{k} C_{k} \eta^{n}\right] .
\end{aligned}
$$

We follow the conventions of Ref. 36: if $\omega=\frac{1}{p !} d x^{\mu_{1}} \wedge \ldots \wedge d x^{\mu_{p}} \omega_{\mu_{1} \ldots \mu_{p}}$, then $\star \omega=\frac{1}{p !(n-p) !} d x^{\mu_{1}}$ $\wedge \ldots \wedge d x^{\mu_{n-p}} \epsilon_{\mu_{1} \ldots m u_{n}} \omega^{\mu_{n-p+1} \ldots \mu_{n}}$, where $\epsilon^{\mu_{1} \ldots \mu_{n}}$ is completely skew symmetric, $\epsilon^{01 \ldots n-1}=1$, and indices are lowered and raised with $\eta_{\mu \nu}=\operatorname{diag}(-1,1, \ldots, 1)$.

The associated infinitesimal deformation of the master action is

$$
S^{(1)}=\int \frac{1}{3 !} \beta_{m n k}\left(B^{m} B^{n} B^{k}+6 \star A^{* m} B^{n} \eta^{k}+3 \star C^{* m} \eta^{n} \eta^{k}\right) .
$$

In particular, $\left(S^{(1)}, S^{(1)}\right)=0$, so the infinitesimal deformation is by itself a complete deformation, $S^{\text {def }}=S+\mu S^{(1)}$. It plays the role of the topological deformation, ${ }^{52,53}$ which has been called "exotic" term in Ref. 10. On the level of the Lie algebra, it amounts to keeping the same inner product but deforming the commutator $\left[l_{m}, l_{n}\right]^{\text {def }}=f_{m n}^{k} l_{k}+\mu \beta_{m n k} l^{* k}$ while keeping all other commutation relations unchanged. That this is legitimate can be checked a posteriori: indeed, the inner product stays invariant because of the complete skew-symmetry of $\beta_{m n k}$, while for the Jacobi identity, only $\left[l_{l},\left[l_{m}, l_{n}\right]^{\mathrm{def}}\right]^{\mathrm{def}}+\operatorname{cyclic}(l, m, n)$ is non-trivial. Evaluating, one finds that this vanishes on account of the cocycle condition since $f_{m n}^{k} \beta_{k l r}+f_{l r}^{k} \beta_{k m n}+\operatorname{cyclic}(l, m, n)$ is completely skew-symmetric in $(l, m, n, r)$.

In degree $(0,3)$, there is no class, because one would need a skew-symmetric three index tensor that is invariant under the coadjoint representation of the Witt algebra. The only candidate is the unique invariant tensor $\delta_{-101}^{m n k}$ under the coadjoint representation of $\mathfrak{s l}(2, \mathbb{R})$, which is no longer invariant under transformations generated by $l_{l}$ with $l \neq-1,0,1$. Note that in the case of $\mathfrak{s}(2, \mathbb{R})$, this is the one that is responsible for the cosmological constant deformation. Whether there are classes in degrees $(1,2)$ and $(2,1)$ needs to be investigated.

Let us now turn to the centrally extended case of the Lie algebra vir $\ltimes \mathfrak{v i r}{ }^{*}$.

First, note that $H^{1}(\mathfrak{v i r})=0=H^{2}(\mathfrak{v i r})$. Indeed, by denoting by $C^{a}$ the ghosts associated to the witt algebra and by $C^{Z}$ the ghost associated to the central element, Chevalley-Eilenberg differential for the Witt algebra is $\gamma=\frac{1}{2} C^{a} C^{b} f_{a b}^{c} \frac{\partial}{\partial C^{c}}$, while the one for the Virasoro algebra is $\gamma^{T}=\frac{1}{2} C^{a} C^{b} f_{a b}^{c} \frac{\partial}{\partial C^{c}}+\omega \frac{\partial}{\partial C^{Z}}$, where $\omega=\omega_{[a b]} C^{a} C^{b}$ is a representative of $H^{2}($ witt), which is 1 dimensional.

For the Virasoro algebra, the cocycle condition in degree 1 for an element $k_{a} C^{a}+k C^{Z}$ implies that $k \omega=-\gamma\left(k_{a} C^{a}\right)$. This implies in turn $k=0$ and, since $H^{1}($ witt $)=0$, that $k_{a} C^{a}$ is $\gamma$ and also $\gamma^{T}$ exact, which gives the result.

The cocycle condition in degree 2 splits depending on whether it involves $C^{Z}$ or not. The former piece implies that the coefficient of $C^{Z}$ is a $\gamma$ cocycle in degree $1 ; H^{1}(\mathrm{witt})=0$ then implies that it is a coboundary, and since there are no coboundaries in degree 1 , that it vanishes. The piece independent of the $C^{Z}$ ghost then has to be a $\gamma^{T}$ and thus also a $\gamma$ cocycle, which implies that it is given by $\omega$ up to a $\gamma$ coboundary $\gamma \eta^{1}$, where $\eta^{1}$ depends on the $C^{a}$ alone, so that $\gamma \eta^{1}=\gamma^{T} \eta_{1}$. But, by construction, $\omega=\gamma^{T} C^{Z}$ which proves the result.

A similar reasoning using in addition that $\omega^{2}$ is a non-trivial cohomology class in $H^{4}$ (witt) then allows one to show that $H^{3}(\mathrm{vir})$ is one dimensional and also described by $\beta$. Indeed, for an element $\alpha=k_{[a b c]} C^{a} C^{b} C^{c}+k_{[a b]} C^{a} C^{B} C^{Z}$, the cocycle condition $\gamma^{T} \alpha=0$ implies $\gamma\left(k_{[a b]} C^{a} C^{B}\right)=0$ so that $k_{[a b]} C^{a} C^{B}=\gamma\left(k_{a} C^{a}\right)+k \omega$. This implies that $\alpha=k_{[a b c]}^{\prime} C^{a} C^{b} C^{c}+\gamma^{T}\left(k_{a} C^{a} C^{Z}\right)+k \omega C^{Z}$, and the cocycle condition becomes $\gamma\left(k_{[a b c]}^{\prime} C^{a} C^{b} C^{c}\right)+k \omega^{2}=0$ which implies $k=0$ and $k_{[a b c]}^{\prime} C^{a} C^{b} C^{c}$ $=k \beta+\gamma\left(l_{[a b]} C^{a} C^{b}\right)=\beta+\gamma^{T}\left(l_{[a b]} C^{a} C^{b}\right)$. Finally, $k \beta=\gamma^{T}\left(l_{[a b]}^{\prime} C^{a} C^{b}+l_{a} C^{a} C^{Z}\right)$ implies $\gamma\left(l_{a} C^{a}\right)$ $=0$ which gives $l_{a} C^{a}=0$ and then $k=0$, so that $\beta$ remains non-trivial. 


\section{DUAL BOUNDARY THEORY}

Following closely the case of $\mathfrak{i s p}_{\mathfrak{n}}(2,1)$ treated in Refs. 42 and 44, the boundary theory when $\mathfrak{s} \mathfrak{D}(2,1) \cong \mathfrak{s l}(2, \mathbb{R})$ is replaced by the Witt or Virasoro algebra is

$$
I[\lambda, \alpha]=\frac{k}{2 \pi} \int d u d \phi\left\langle\dot{\lambda} \lambda^{-1}, \alpha^{\prime}\right\rangle,
$$

where $\lambda(u, \phi)$ is a map from the cylinder to the $\operatorname{Diff}\left(S^{1}\right) /$ Virasoro group, $\alpha(u, \phi)$ a map whose value is an associated coadjoint vector, and the inner product $\langle\cdot, \cdot\rangle$ between coadjoint vectors and Lie algebra elements replaces $2 \operatorname{Tr}$ in Ref. 44 . Note that in that reference, the integrand contains an additional term $-\operatorname{Tr}\left(\lambda_{\mathrm{SL}(2, \mathbb{R})}^{\prime} \lambda_{\mathrm{SL}(2, \mathbb{R})}^{-1}\right)^{2}$ originating from a Gibbons-Hawking type improvement term needed to account for the non- trivial asymptotics of the spin connection. Because of the absence of an invariant trace, this term cannot be extended to a $\operatorname{Diff}\left(S^{1}\right) /$ Virasoro group element. In order to continue to describe an extension of gravity and include the $u$-dependent solutions discussed at the end of Section IV, one should add this terms, with the group element restricted to $\operatorname{SL}(2, \mathbb{R})$. For simplicity, we choose not to do so here and concentrate on the model defined by (6.1).

The equations of motion of the model are

$$
\left(\dot{\lambda} \lambda^{-1}\right)^{\prime}=0, \quad D_{u}^{-\dot{\lambda} \lambda^{-1}} \alpha^{\prime}=0 .
$$

The general solution involves a factorized group element $\lambda=\mu(u) v(\phi)$ and $\alpha=\mu(\rho(\phi)+\delta(u)) \mu^{-1}$. The gauge invariance of the action is $\lambda \rightarrow v(u) \lambda, \alpha \rightarrow v \alpha v^{-1}$, while the global symmetries are $\lambda \rightarrow \lambda \Theta(\phi)^{-1}, \alpha \rightarrow \alpha+\lambda \Sigma(\phi) \lambda^{-1}$. The infinitesimal versions of the latter are $\delta_{\theta} \lambda=-\lambda \theta, \delta_{\theta} \alpha=0$, and $\delta_{\sigma} \lambda=0, \delta_{\sigma} \alpha=\lambda \sigma \lambda^{-1}$ with Noether currents

$$
\begin{gathered}
J_{\theta}^{0}=\langle\theta, J\rangle, \quad J=-\frac{k}{2 \pi}\left[\lambda^{-1} \alpha^{\prime} \lambda\right], \quad J_{\theta}^{1}=0, \\
P_{\sigma}^{0}=\langle\sigma, P\rangle, \quad P=\frac{k}{2 \pi} \lambda^{-1} \lambda^{\prime}, \quad P_{\sigma}^{1}=0 .
\end{gathered}
$$

In terms of generators, $J_{m}=\left\langle l_{m}, J\right\rangle, \zeta=\langle Z, J\rangle$, and $P^{m}=\left\langle l^{* m}, P\right\rangle, \zeta^{*}=\left\langle Z^{*}, P\right\rangle$, one then reads off the current algebra from the structure constants and the inner product,

$$
\begin{aligned}
& \left\{P^{m}(\phi), P^{n}\left(\phi^{\prime}\right)\right\}^{*}=0=\left\{P^{m}(\phi), \zeta\left(\phi^{\prime}\right)\right\}^{*}=\left\{P^{m}(\phi), \zeta^{*}\left(\phi^{\prime}\right)\right\}^{*}, \\
& \left\{\zeta(\phi), \zeta^{*}\left(\phi^{\prime}\right)\right\}^{*}=-\frac{k}{2 \pi} \partial_{\phi} \delta\left(\phi-\phi^{\prime}\right), \quad\left\{\zeta(\phi), J_{m}\left(\phi^{\prime}\right)\right\}^{*}=0, \\
& \left\{J_{m}(\phi), \zeta^{*}\left(\phi^{\prime}\right)\right\}^{*}=-\frac{1}{12} m\left(m^{2}-1\right) P^{-m}(\phi) \delta\left(\phi-\phi^{\prime}\right), \\
& \left\{J_{m}(\phi), P^{n}\left(\phi^{\prime}\right)\right\}^{*}=(n-2 m) P^{n-m}(\phi) \delta\left(\phi-\phi^{\prime}\right)-\frac{k}{2 \pi} \delta_{m}^{n} \partial_{\phi} \delta\left(\phi-\phi^{\prime}\right), \\
& \left\{J_{m}(\phi), J_{n}\left(\phi^{\prime}\right)\right\}^{*}=\left[(m-n) J_{m+n}(\phi)+\frac{\zeta(\phi)}{12} \delta_{m+n}^{0} m\left(m^{2}-1\right)\right] \delta\left(\phi-\phi^{\prime}\right) .
\end{aligned}
$$

The natural combination that is available for a classical Sugawara construction is

$$
\mathcal{P} \approx-\frac{2 \pi}{k}\left(J_{m} P^{m}+\zeta \zeta^{*}\right)
$$

and

$$
\left\{\mathcal{P}(\phi), \mathcal{P}\left(\phi^{\prime}\right)\right\}^{*}=\left(\mathcal{P}(\phi)+\mathcal{P}\left(\phi^{\prime}\right)\right) \partial_{\phi} \delta\left(\phi-\phi^{\prime}\right),
$$

or, in terms of modes $\mathcal{J}_{m}=\int_{0}^{2 \pi} d \phi e^{i m \phi \mathcal{P}}$,

$$
i\left\{\mathcal{J}_{m}, \mathcal{J}_{n}\right\}=(m-n) \mathcal{J}_{m+n},
$$

which expresses conformal invariance of the model. 


\section{ACKNOWLEDGMENTS}

This work is supported by two FNRS/MINCyT collaboration agreements. The work of G.B. and G.G. is partially funded by the Fund for Scientific Research-FNRS Belgium (convention FRFC PDR T.1025.14 and convention IISN 4.4503.15) and by "Communauté française de Belgique - Actions de Recherche Concertées." The work of G.G. is also supported by a donation from the Solvay family.

${ }^{1}$ M. Blencowe, "A consistent interacting massless higher spin field theory in d = (2+1)," Classical Quantum Gravity 6, 443 (1989).

${ }^{2}$ M. Henneaux and S.-J. Rey, "Nonlinear $W_{\text {infinity }}$ as asymptotic symmetry of three-dimensional higher spin Anti-de Sitter gravity," JHEP 1012, 007 (2010); e-print arXiv:1008.4579.

3 A. Campoleoni, S. Fredenhagen, S. Pfenninger, and S. Theisen, "Asymptotic symmetries of three-dimensional gravity coupled to higher-spin fields," JHEP 1011, 007 (2010); e-print arXiv:1008.4744.

${ }^{4}$ M. R. Gaberdiel, R. Gopakumar, and A. Saha, "Quantum $W$-symmetry in $A d S_{3}$," JHEP 2011, 1; e-print arXiv:1009.6087.

${ }^{5}$ M. R. Gaberdiel and R. Gopakumar, "An AdS3 dual for minimal model CFTs," Phys. Rev. D 83, 066007 (2011); e-print arXiv:1011.2986.

${ }^{6}$ M. R. Gaberdiel and R. Gopakumar, "Minimal model holography,” J. Phys. A: Math. Theor. 46, 214002 (2013); e-print arXiv:1207.6697.

${ }^{7}$ H. Afshar, A. Bagchi, R. Fareghbal, D. Grumiller, and J. Rosseel, "Higher spin theory in 3-dimensional flat space," Phys. Rev. Lett. 111, 121603 (2013); e-print arXiv:1307.4768.

${ }^{8}$ H. A. Gonzalez, J. Matulich, M. Pino, and R. Troncoso, "Asymptotically flat spacetimes in three-dimensional higher spin gravity," JHEP 1309, 016 (2013); e-print arXiv:1307.5651.

9 A. Achucarro and P. K. Townsend, "A Chern-Simons action for three-dimensional Anti-de Sitter supergravity theories," Phys. Lett. B 180, 89 (1986).

${ }^{10}$ E. Witten, "(2+1)-dimensional gravity as an exactly soluble system,” Nucl. Phys. B 311, 46 (1988).

${ }^{11}$ A. Ashtekar and J. D. Romano, "Chern-Simons and Palatini actions and (2+1) gravity," Phys. Lett. B 229, 56-60 (1989).

12 J. D. Romano, "Geometrodynamics versus connection dynamics (in the context of $(2+1)$ and $(3+1)$ gravity," Gen. Relativ. Gravitation 25, 759-854 (1993); e-print arXiv:gr-qc/9303032.

${ }^{13}$ E. Witten, "Coadjoint orbits of the Virasoro group," Commun. Math. Phys. 114, 1 (1988).

${ }^{14}$ I. Bakas, "Conformal invariance, the KdV equation and coadjoint orbits of the Virasoro algebra," Nucl. Phys. B 302, 189-203 (1988).

15 W. Taylor, "Virasoro representations on diff S1 / S1 coadjoint orbits," e-print arXiv:hep-th/9204091.

${ }^{16}$ J. Balog, L. Feher, and L. Palla, "Coadjoint orbits of the Virasoro algebra and the global Liouville equation," Int. J. Mod. Phys. A 13, 315-362 (1998); e-print arXiv:hep-th/9703045.

${ }^{17}$ N. Ikeda, "Two-dimensional gravity and nonlinear gauge theory," Ann. Phys. 235, 435-464 (1994); e-print arXiv:hep-th/ 9312059.

${ }^{18}$ P. Schaller and T. Strobl, "Poisson structure induced (topological) field theories," Mod. Phys. Lett. A 9, 3129-3136 (1994); e-print arXiv:hep-th/9405110.

${ }^{19}$ M. Kontsevich, "Topics in algebra-deformation theory," Lectures Notes (1994).

${ }^{20}$ A. S. Cattaneo and G. Felder, "A path integral approach to the Kontsevich quantization formula," Commun. Math. Phys. 212, 591-611 (2000); e-print arXiv:math/9902090.

${ }^{21}$ A. Ashtekar, J. Bicak, and B. G. Schmidt, “Asymptotic structure of symmetry-reduced general relativity," Phys. Rev. D 55, 669-686 (1997).

${ }^{22}$ G. Barnich and G. Compère, "Classical central extension for asymptotic symmetries at null infinity in three spacetime dimensions," Classical Quantum Gravity 24, F15 (2007); e-print arXiv:gr-qc/0610130; Corrigendum, 24, 3139 (2007).

${ }^{23}$ A. O. Barut and R. Raczka, Theory of Group Representations and Applications (Polish Scientific Publishers, Warszawa, 1980).

${ }^{24}$ G. Barnich and B. Oblak, "Notes on the BMS group in three dimensions: I. Induced representations," JHEP 1406, 129 (2014); e-print arXiv:1403.5803.

${ }^{25}$ A. Garbarz and M. Leston, "Classification of boundary gravitons in $\mathrm{AdS}_{3}$ gravity," JHEP 1405, 141 (2014); e-print arXiv: 1403.3367.

${ }^{26}$ G. Barnich and B. Oblak, "Holographic positive energy theorems in three-dimensional gravity," Classical Quantum Gravity 31, 152001 (2014); e-print arXiv:1403.3835.

${ }^{27}$ G. Barnich and B. Oblak, "Notes on the BMS group in three dimensions: II. Coadjoint representation,” JHEP 1503, 033 (2015); e-print arXiv:1502.00010.

${ }^{28}$ I. A. Batalin and G. A. Vilkovisky, "Gauge algebra and quantization," Phys. Lett. B 102, 27-31 (1981).

${ }^{29}$ I. A. Batalin and G. A. Vilkovisky, "Feynman rules for reducible gauge theories," Phys. Lett. B 120, 166-170 (1983).

${ }^{30}$ I. A. Batalin and G. A. Vilkovisky, "Quantization of gauge theories with linearly dependent generators," Phys. Rev. D 28, 2567-2582 (1983).

${ }^{31}$ I. A. Batalin and G. A. Vilkovisky, "Closure of the gauge algebra, generalized Lie equations and Feynman rules," Nucl. Phys. B 234, 106-124 (1984).

${ }^{32}$ I. A. Batalin and G. A. Vilkovisky, "Existence theorem for gauge algebra," J. Math. Phys. 26, 172-184 (1985).

${ }^{33}$ M. Henneaux and C. Teitelboim, Quantization of Gauge Systems (Princeton University Press, 1992).

${ }^{34}$ J. Gomis, J. París, and S. Samuel, "Antibracket, antifields and gauge theory quantization,” Phys. Rep. 259, 1-145 (1995); e-print arXiv:hep-th/9412228.

${ }^{35} \mathrm{G}$. Barnich and M. Henneaux, "Consistent couplings between fields with a gauge freedom and deformations of the master equation,” Phys. Lett. B 311, 123-129 (1993); e-print arXiv:hep-th/9304057. 
${ }^{36}$ G. Barnich, F. Brandt, and M. Henneaux, "Local BRST cohomology in gauge theories," Phys. Rep. 338, 439-569 (2000); e-print arXiv:hep-th/0002245.

${ }^{37}$ A. Barkallil, G. Barnich, and C. Schomblond, "Results on the Wess-Zumino consistency condition for arbitrary Lie algebras," J. Math. Phys. 43, 5987-6015 (2002); e-print arXiv:math-ph/0205047.

${ }^{38}$ M. Alexandrov, M. Kontsevich, A. Schwartz, and O. Zaboronsky, "The geometry of the master equation and topological quantum field theory," Int. J. Mod. Phys. A 12, 1405-1430 (1997); e-print arXiv:hep-th/9502010.

${ }^{39}$ G. Barnich and M. Grigoriev, “A Poincare lemma for sigma models of AKSZ type,” J. Geom. Phys. 61, 663-674 (2011); e-print arXiv:0905.0547.

${ }^{40}$ E. Witten, "Quantum field theory and the Jones polynomial," Commun. Math. Phys. 121, 351 (1989)

${ }^{41}$ S. Elitzur, G. W. Moore, A. Schwimmer, and N. Seiberg, "Remarks on the canonical quantization of the Chern-SimonsWitten theory," Nucl. Phys. B 326, 108 (1989).

${ }^{42}$ P. Salomonson, B. Skagerstam, and A. Stern, "ISO(2,1) chiral models and quantum gravity in (2+1)-dimensions," Nucl. Phys. B 347, 769-782 (1990).

${ }^{43}$ O. Coussaert, M. Henneaux, and P. van Driel, "The asymptotic dynamics of three-dimensional Einstein gravity with a negative cosmological constant," Classical Quantum Gravity 12, 2961-2966 (1995); e-print arXiv:gr-qc/9506019.

${ }^{44}$ G. Barnich and H. A. Gonzalez, "Dual dynamics of three dimensional asymptotically flat Einstein gravity at null infinity," JHEP 1305, 016 (2013); e-print arXiv:1303.1075.

${ }^{45}$ O. Hohm, "On the infinite-dimensional spin-2 symmetries in Kaluza-Klein theories," Phys. Rev. D 73, 044003 (2006); e-print arXiv:hep-th/0511165.

${ }^{46}$ O. Hohm, "Massive Kaluza-Klein theories and their spontaneously broken symmetries," e-print arXiv:hep-th/0612235.

${ }^{47}$ V. Aldaya and J. Navarro-Salas, "Quantization on the Virasoro group," Commun. Math. Phys. 126, 575 (1989).

${ }^{48}$ G. Barnich and C. Troessaert, “Aspects of the BMS/CFT correspondence,” JHEP 05, 062 (2010); e-print arXiv: 1001.1541.

${ }^{49}$ G. Hochschild and J. Serre, "Cohomology of Lie algebras," Ann. Math. 57(3), 591 (1953).

${ }^{50}$ I. M. Gel'fand and D. B. Fuks, “The cohomologies of the Lie algebra of the vector fields in a circle," Funct. Anal. Its Appl. 2, 342-343 (1968).

${ }^{51}$ D. Fuks, Cohomology of Infinite-Dimensional Lie Algebras (Consultants Bureau, New York, 1986).

52 S. Deser, R. Jackiw, and S. Templeton, "Three-dimensional massive gauge theories," Phys. Rev. Lett. 48, 975-978 (1982).

${ }^{53}$ S. Deser, R. Jackiw, and S. Templeton, "Topologically massive gauge theories," Ann. Phys. 140, $372-411$ (1982). 